\title{
Daniel Duband : cinquante ans de contributions scientifiques à l'hydrologie (1962-2011)
}

\author{
Charles OBLED ${ }^{1}$
}

1. Professeur émérite à l'INP-Grenoble, Laboratoire LTHE

\begin{abstract}
RÉSUMÉ. - Cette communication présente les principales contributions scientifiques apportées par Daniel Duband à l'hydrologie, pendant 50 ans. Il a été notamment à l'origine d'avancées dans trois domaines : la prédétermination des crues extrêmes avec la méthode du Gradex, la prévision probabiliste des précipitations par une approche statistique d'analogie, et la modélisation hydrologique avec la méthode DPFT. L'article retrace la genèse de ces méthodes et leur devenir dans la communauté hydrologique.
\end{abstract}

Mots-clés : Daniel Duband, Gradex, analogues, DPFT

\section{Daniel Duband: fifty years of scientific contributions to hydrology (1962-2011)}

\begin{abstract}
This paper is a tribute to the main scientific advances carried out by Daniel Duband to hydrology. A focus is presented on three domains: flood frequency analysis with the Gradex method, probabilistic quantitative forecasting on rainfall by a statistical method based on analogue situations, and rainfall-runoff modelling with the ERUDHIT method. The paper gives explanations on the genesis of each method and their dissemination within the hydrological community.
\end{abstract}

Key-words: Daniel Duband, Gradex, analogues, ERUDHIT

\section{AVERTISSEMENT PRÉLIMINAIRE}

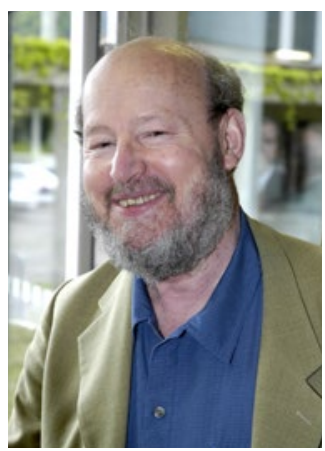

D. Duband

(Photo Ph. Bois)
Retracer les apports scientifiques de Daniel Duband n'est pas chose simple, tant sont nombreux les aspects de l'Hydrologie auxquels il a contribué. Compte tenu de ce que personnellement je lui dois, il m'était difficile de décliner cette demande, tout à la fois honorifique mais aussi redoutable. En effet, je m'aperçois que je l'ai côtoyé dans les moments les plus créatifs de sa carrière, qu'il m'a accordé son estime et beaucoup de son temps, et il ne faut pas aujourd'hui que je trahisse cette confiance. Pourtant, ce qui suit s'appuiera en partie sur des souvenirs qui pour certains s'estompent, sont incomplets ou comportent une part de subjectivité que l'on voudra bien, j'espère, me pardonner ...

\section{INTRODUCTION}

Comme on le sait, la carrière scientifique de Daniel Duband s'est déroulée presqu'entièrement au sein d'Electricité de France. Mais il n'est peut-être pas inutile de rappeler ce qu'était le contexte qu'il a trouvé à son arrivée dans cette grande entreprise publique, créée à la Libération sur proposition du Conseil National de la Résistance...

Dans les années 1950, l'Hydraulique battait son plein, et il fallait optimiser l'exploitation des grands réservoirs saisonniers que l'on venait de construire ou dont on avait reçu la concession. Et pour ce faire, les prévisions statistiques sont apparues, à EdF, comme l'outil privilégié permettant de piloter efficacement leur gestion. Cette ligne directrice remontait en fait à la fin des années 1940, avec les contributions d'André Coutagne (1948), mais surtout de Stéphane Ferry et Fernand Lugiez (cf. Ferry, 1949 ; Ferry et Lugiez, 1951 ; Lugiez, 1954 ; Ferry, 1955). Ces publications sont notamment consacrées à la prévision, en montagne, des apports de fonte nivale (pour des barrages comme Tignes, mis en eau en 1953, mais aussi du Chambon, achevé en 1935 ...). Un premier bilan de dix ans de prévision statistique à EDF est présenté lors de l'Assemblée générale de l'UGGI à Helsinki (Lugiez et Guillot, 1960). Pierre Guillot va ensuite jouer un rôle important dans la carrière et les réalisations de D. Duband. Lui-même est entré à EdF en 1952 au Groupe Régional de Production Hydraulique Massif Central et a contribué aux études générales pour le barrage de Bort les Orgues (mis en eau en 1952). Il dépend alors de la Direction de la Production Hydraulique, qui dispose d'un service d'appui national (bien que basé à Grenoble ...!): la Division Technique Générale de la Production Hydraulique. Son premier directeur, S. Ferry, la dirige de Paris depuis 1949, mais son adjoint F. Lugiez, basé à Grenoble, en devient la cheville ouvrière dès 1952. P. Guillot le rejoint en 1958, et s'implique alors dans la prévision hydrologique et la prédétermination des débits de crues de projet. De formation généraliste (ingénieur Ponts et Chaussées), il a vite pris conscience de la nécessité de renforcer le potentiel théorique et le savoir-faire en probabilités et en statistiques de son équipe. Certes, il existe bien à EdF une Direction des Etudes et Recherches à laquelle il pourrait faire appel, mais celle-ci est à Chatou, loin de Grenoble et surtout, à ses yeux, un peu loin des réalités opérationnelles de la production ... 
En effet, comme il le synthétisera dans un rapport sur la prévision hydrologique (Guillot, 1969), les besoins concrets pour la production hydraulique s'articulent autour de :

- la prévision des apports mensuels et saisonniers ;

- la prévision des étiages et l'évaluation de la probabilité des débits les plus faibles ;

- La prévision des crues ordinaires et des débits à court terme à partir des pluies ;

- l'évaluation de la probabilité des crues extrêmes.

Les chapitres I et III font surtout appel aux techniques de corrélation multiple, et les chapitres II et IV aux modèles probabilistes, notamment des valeurs extrêmes. C'est pourquoi il obtient, pour le seconder, l'affectation d'un jeune universitaire prometteur : Daniel Duband, sorti en 1959 de l'ISUP (Institut de Statistiques de l'Université de Paris) avec le Certificat d'Etudes Supérieures en Statistiques, entré à EDF en 1962, où il est d'abord affecté à l'échelon DTG de Brive. Il a de solides bases en probabilités et statistiques, mais curieusement, sa première apparition comme auteur dans une publication (Lugiez et al., 1963) concernera la prise en compte de la capacité de rétention du sol pour la prévision des débits à court terme, un sujet qui fleure plutôt l'hydrologie physique ... Peu après, en 1966, D. Duband rejoindra P. Guillot au siège de la DTG à Grenoble, alors dirigée par son «père fondateur » Fernand Lugiez. Et s'ensuivra une phase extrêmement riche de production à la fois de haut niveau scientifique mais aussi très immédiatement appliquée.

\section{LA METHODE DU GRADEX}

Le problème des crues extrêmes (en fait des crues « de projet ») préoccupait les ingénieurs depuis longtemps. Etienne Halphen, comme le rappelle Morlat (1956), avait dès 1940 proposé une série de lois pour l'étude des débits. D'autres publications d'ingénieurs d'EDF suivront : Morlat (1951), Bernier (1956 et 1959). Pierre Guillot commence d'ailleurs par suivre cette voie consistant à travailler sur les seules séries de débits (Guillot, 1963) ... Mais le doute s'insinue quant à l'option de travailler sur les seules séries de débits ... A l'époque, le dimensionnement de l'évacuateur de crue de Serre-Ponçon (mis en eau en 1961) avait suscité un grand débat ... Premier grand ouvrage situé dans les Alpes du Sud, région sujette aux séismes, on avait choisi de le construire en terre, donc très vulnérable à un risque de surverse ... Un bon exemple de ces réflexions est l'article de Labaye (1956). Et finalement, la crue de projet avait été fixée à la tri-millénnale, contrairement à la millénnale retenue à l'époque pour les barrages en béton. Or avec la série de maxima annuels disponible, et selon que l'on choisissait Gumbel ou Fréchet pour l'ajustement, le débit de projet passait de 3000 à quasiment $12000 \mathrm{~m} 3 / \mathrm{s}$... !

Et c'est là que P. Guillot et $\mathrm{D}$. Duband proposeront une intuition déterminante : en considérant que, quand on va vers des débits aussi exceptionnels, la part de rétention des sols prélevée sur le volume de pluie tombé devient secondaire, et c'est alors la distribution de ces volumes de pluie qui contrôle la queue de la distribution des volumes de ces débits extrêmes ... Il y a d'ailleurs une analogie très forte entre ce concept et celui développé par le modèle hydrologique « simplifié »du Soil Conservation Service (SCS 1972 et 1973). Cela se voit déjà sur le graphique des faisceaux de courbes illustrant les «Curve Numbers » (Fig. 1) qui ressemblent étrangement au diagramme que proposent Guillot et Duband pour illustrer la distribution de probabilité des rétentions (Fig. 2) ... Mais curieusement, ils ne feront jamais allusion ni référence explicite à cette similitude de concept. C'est pourtant un modèle hydrologique beaucoup plus riche qu'on ne le croit, et qu'ils ont implicitement utilisé. Il montre notamment l'inanité du reproche parfois fait à la méthode du Gradex qui considèrerait qu'au delà d'un certain seuil, tout supplément de la pluie tombée deviendrait à $100 \%$ efficace : c'est une vision caricaturale qui n'est pas nécessaire pour justifier la méthode !

Cependant, l'idée première est que la distribution des volumes écoulés provient de la soustraction de deux

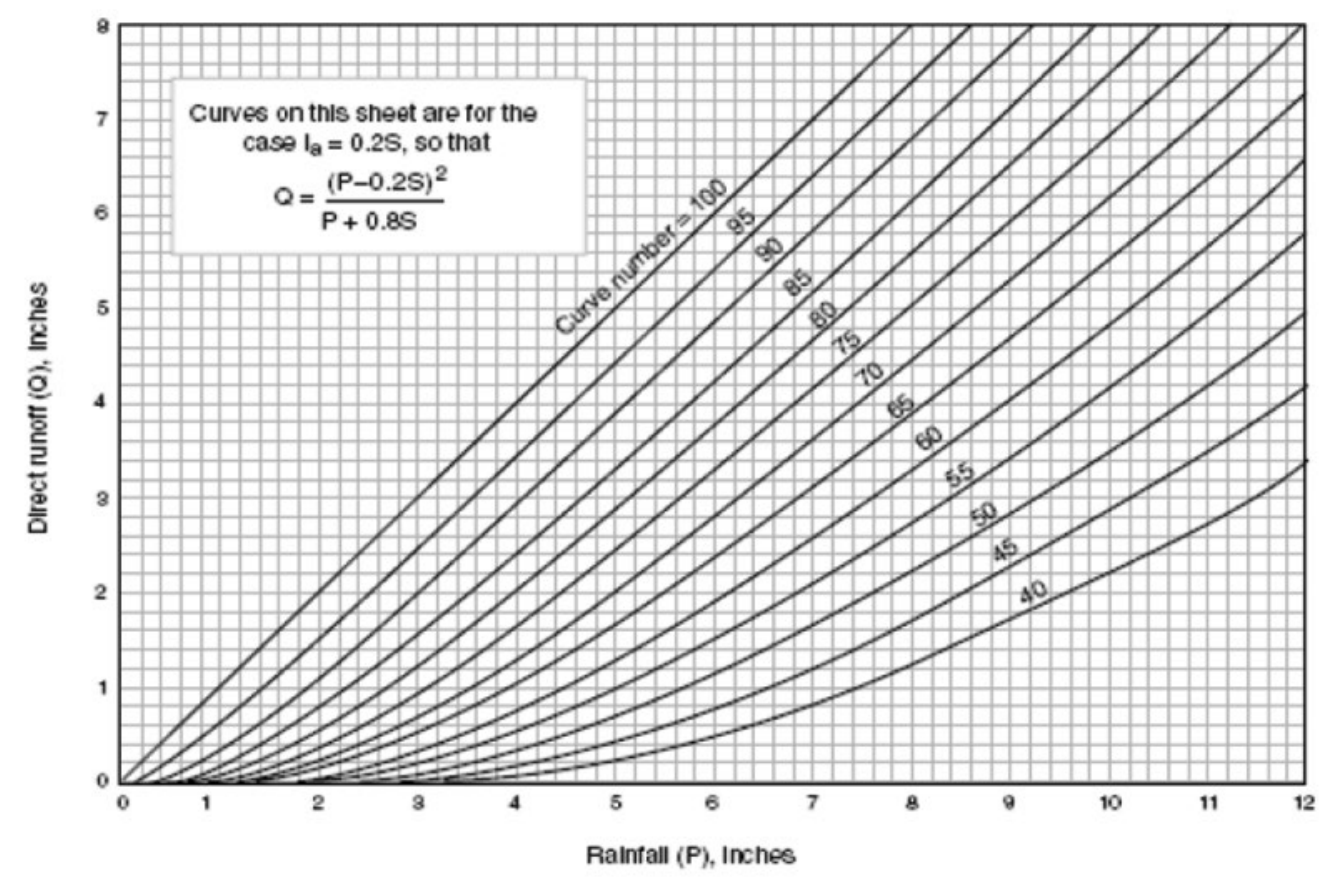

Figure 1 : Diagramme des "Curve Number " du modèle SCS reliant les précipitations et les écoulements en fonction de la rétention potentielle $S=(1000 / C N)-10$ (extrait de SCS, 1972). 


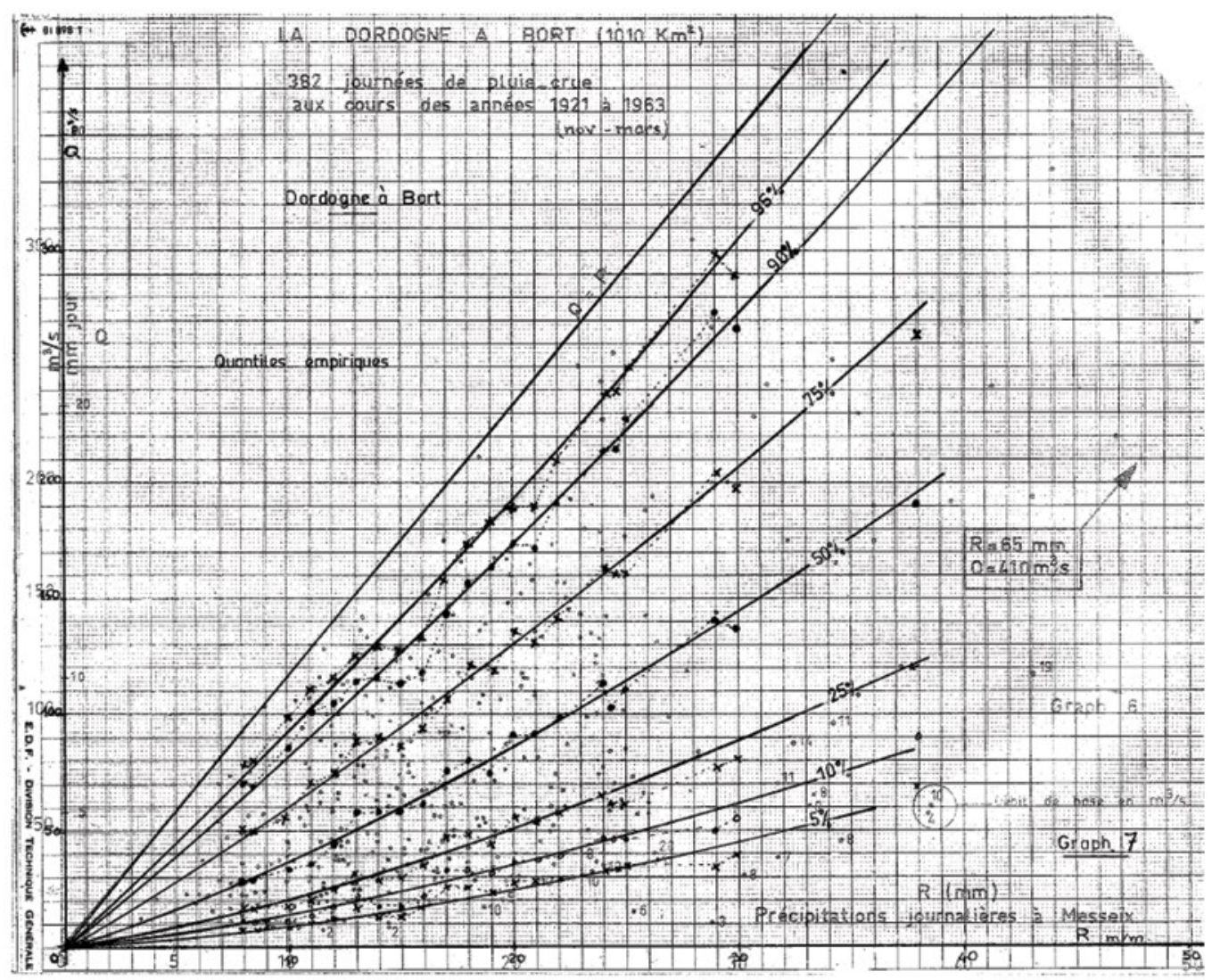

Figure 2 : Relation entre les précipitations et les écoulements de crue pour 382 événements et superposition sur calque des courbes quantiliques empiriques de la rétention (extrait de P. Guillot et D. Duband-communication SHF-commission des débits -Avril 1966).

variables aléatoires indépendantes (i.e. le volume de pluie tombé moins le volume de rétention), considérés sur la durée (moyenne) de l'événement de crue, et que, dans les grandes valeurs, cette variable pluie devient prédominante ... Cette idée revient bien à P. Guillot et D. Duband. Ensuite, le choix d'une loi particulière pour les pluies extrêmes, ici la loi de Gumbel, constitue une deuxième hypothèse, avec des argumentations qui ont d'ailleurs évolué dans le temps. $\mathrm{Au}$ début, on s'appuyait sur la récente théorie des Valeurs Extrêmes proposée par Gumbel $(1954,1956)$. Ce mathématicien allemand, qui a séjourné en France de 1932 à 1940, avant de fuir aux Etats-Unis, a d'ailleurs travaillé avec Borel et Fréchet et publié dans la Houille Blanche. En supposant la distribution des pluies journalières exponentielle, alors la distribution des maxima annuels qui en dérive est gumbélienne, et l'on appelle gradex (gradient de l'exponentielle), la pente de la droite d'ajustement dans le diagramme fonctionnel de Gumbel. La " méthode du Gradex » rassemble, elle, les différents concepts et choix de lois ci-dessus. Elle consiste pour l'essentiel à extrapoler, dans un diagramme de Gumbel, la distribution des volumes de crues selon une droite de même pente, et donc parallèle à la distribution des pluies (Fig. 3). Elle a été présentée pour la première fois en 1967 au Symposium International d'Hydrologie de Fort Collins (Guillot et Duband, 1967).

Or ce symposium fut certainement un tournant dans la vie scientifique de D. Duband, puisqu'il y a présenté deux autres communications : l'une avec P. Guillot sur « le calcul des probabilités d'étiage prolongé à partir des corrélations entre pluies et débits ", et une autre comme seul auteur sur « La composition des lois de probabilités de pluies journalières ». De plus, ce symposium était organisé par un statisticien d'origine yougoslave, installé à Fort Collins depuis 1960, mais diplômé, en 1939, de l'Ecole des Ingénieurs Hydrauliciens de Grenoble : V. Yevjevitch ... Et enfin, toujours en cette année 1967, Grenoble voit le départ en retraite de Maurice Pardé, hydrologue et potamologue mondialement connu, et Professeur à l'Ecole des Ingénieurs Hydrauliciens. Pour compenser ce départ, l'Ecole demandera alors à P. Guillot d'y assurer désormais un cours d'Hydrologie Statistique, dans lequel, dès 1968, il entraînera D. Duband.

Ici, il nous faut faire un autre petit aparté pour rappeler aussi le contexte de l'époque. Comme on l'a évoqué, la gestion des grands ouvrages récemment achevés avait nécessité des modèles efficaces de prévision des ressources (apports à Serre Ponçon par exemple), d'où une énorme pratique de la corrélation multiple et de l'interprétation des corrélations partielles pour la sélection de variables prédictives, essentiellement à partir des tests mis au point par l'école anglaise de statistique. A l'époque, d'ailleurs, le livre de chevet de D. Duband est celui de Kendall et Stuart (1963), " la Bible des statisticiens ", soit plus de mille pages en trois tomes grand format ... et notamment le tome 2 « Inference and Relationship »...

Mais il faut aussi rappeler le contexte technologique, et notamment les moyens de calcul existants. Jusqu'en 1965, on a utilisé surtout des machines à calculer manuelles, puis électriques (les fameuses "Monroe ", dont le large chariot permettait, en mettant la valeur de $\mathrm{X}$ cadrée à droite, celle de $\mathrm{Y}$ cadrée tout à gauche et en élevant au carré, de retrouver distinctement $\mathrm{X}^{2}$ à droite, $\mathrm{Y}^{2}$ à gauche, et 2.X.Y au milieu, que l'on sommait ensuite sur chaque couple $\mathrm{Xi}$, 


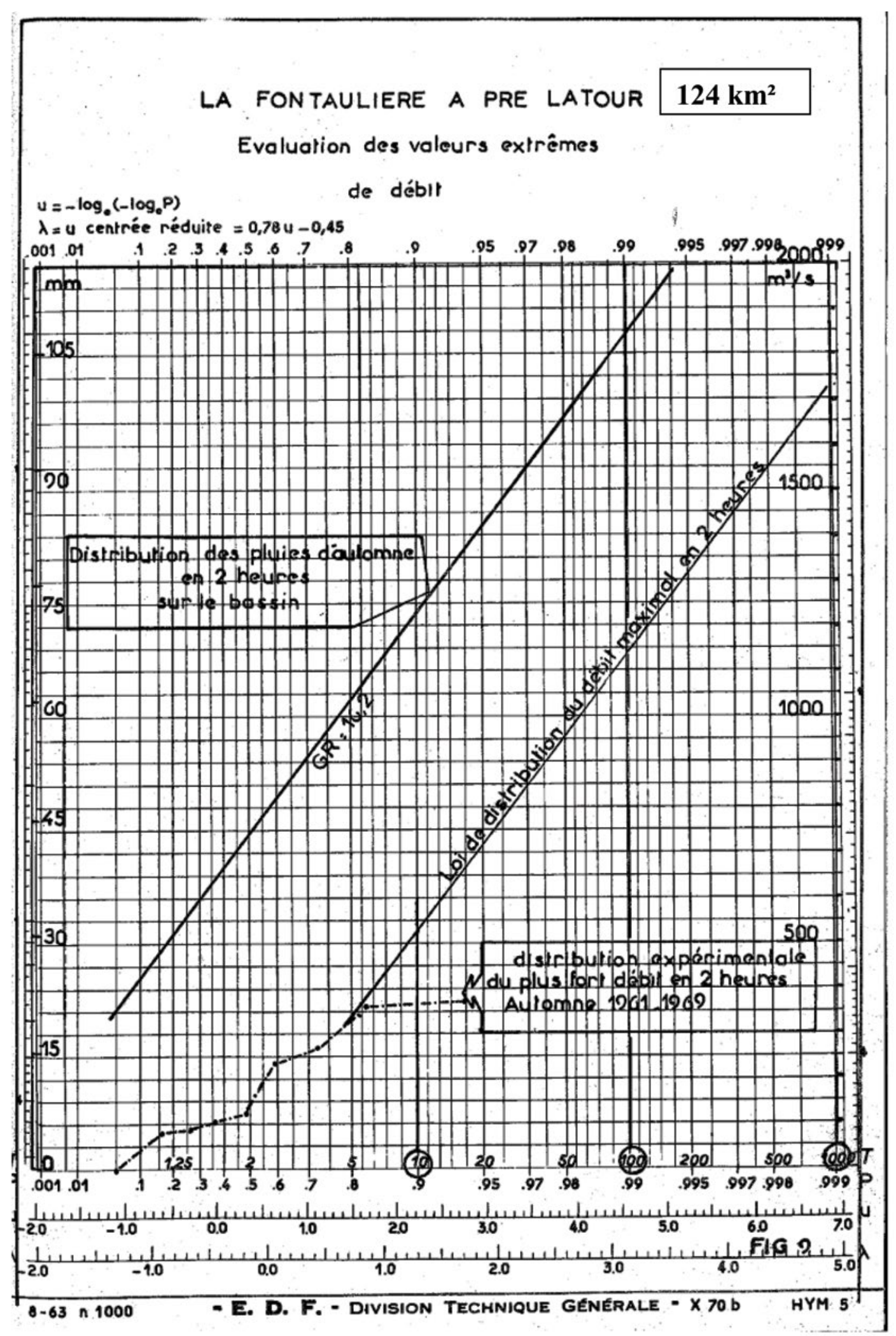

Figure 3 : Exemple d'application du Gradex (D .Duband- Note interne DTG 1971).

Yi successivement). Mais même avec ces astuces, la moindre corrélation prenait des heures, voire des jours quand on voulait tester de nombreuses combinaisons de prédicteurs ...

Or en 1965 la DTG voit apparaître, à la demande justement du Service Hydrométéorologie, son premier calculateur, une CAE 510 (fabriquée par la Compagnie Européenne d'Automatisme et de Electronique, filiale de la CSF créée en 1960), disposant d'un lecteur perforateur de ruban, d'un dérouleur de bande magnétique, et pour sortie papier d'une simple machine à écrire, mais avec un interpréteur du récent langage ALGOL 60 qui permettait de coder très proprement des algorithmes scientifiques ... Il faudra attendre 1969 pour que la DTG s'équipe d'un ordinateur beaucoup plus performant de marque ICL 1902 A (International 
Computer Limited), avec lecteur de ruban perforé, cartes perforées, bandes et disques magnétiques, une vraie imprimante ... Le langage de programmation est toujours l'ALGOL 60, mais le système d'exploitation permet désormais de gérer plusieurs tâches ou programmes qui s'enchaînent. Et l'un des premiers, REMULOB, concerne évidemment la régression multiple avec sélection de variables et diverses options de transformation de ces dernières. Avec désormais un vrai service informatique, doté d'un ingénieur spécialisé, de deux opérateurs et d'une programmeuse scientifique, des calculs bien plus volumineux, inimaginables avec des machines de bureau, devenaient désormais possibles ...

Or on se rappelle que parmi les besoins listés par Guillot (1969) figurait aussi « la prévision des crues ordinaires et des débits à court terme à partir des pluies »... Et le vrai problème pour les besoins de la Production Hydraulique et les Mouvements d'Energie était d'étendre les échéances en essayant d'anticiper les pluies, donc de prévoir les pluies futures sur les bassins concernés ...

En cette fin des années 1960, les modèles mathématiques en météo commençaient certes à proposer des prévisions de champs de pression, mais en ce qui concernait les précipitations, on utilisait encore à des résolutions bien trop grandes, sans parler de la paramétrisation rustique utilisée dans ces modèles pour les processus précipitants .... Donc c'étaient des prévisionnistes de la Météorologie Nationale, détachés à EdF dans les centres de prévision de Grenoble, Lyon, Brive et Toulouse, qui assuraient ces prévisions de précipitations, les plus quantitatives possibles ....

Des tentatives avaient bien été faites aux Etats-Unis pour tenter de relier les précipitations (mesurées à des stations) avec des paramètres aérologiques ou synoptiques, mais les performances étaient relativement médiocres. En effet, il s'agissait de modèles linéaires mais calés (là encore par corrélation multiple) sur toutes les journées quel que soit leur type de temps, d'où une trop grande rigidité. Il aurait fallu quelque chose de plus flexible, de plus dynamique, modulé selon la circulation atmosphérique ...

Des tentatives avaient eu lieu, dans les années 1950 (par M. Fontaine, à la direction de la Climatologie) consistant, pour un jour donné dont on venait de prévoir la situation, à rechercher manuellement parmi des cartes de situations passées celles qui ressemblaient à la situation analysée, et à considérer les pluies recueillies lors de ces journées passées ... Mais le côté manuel et subjectif n'avait pas permis d'aller très loin ... D. Duband, avec sa formation en analyse des données, et avec l'aide des prévisionnistes susmentionnés avec qui il collabore quotidiennement, décide alors de s'attaquer au problème de manière plus systématique et objective.

\section{LA METHODE DES ANALOGUES}

Un premier consensus était nécessaire quant aux sources de données à utiliser. Il faut évidemment des données en altitude : ce seront donc les radiosondages. Et sur quel domaine? Il s'agira de l'Europe de l'Ouest, l'Afrique du Nord et l'Atlantique. Pour cette dernière zone, à l'époque, les radiosondages étaient assurés par des corvettes météo, appartenant à différents pays, et tournant en rond autour de points prédéfinis $\mathrm{A}, \mathrm{C}, \mathrm{I}, \mathrm{J}, \mathrm{K}$ et où elles passaient à heures fixes faire le lâcher de ballon et l'observation.

Un premier balayage a conduit à retenir 25 radiosondages (Fig. 4), et à privilégier le niveau $700 \mathrm{mb}$ (car à l'époque, on ne dit pas encore $\mathrm{hPa}$ !). On complète cela par un réseau de 11 stations de pression au sol, plus restreint et plus dense surtout sur le territoire métropolitain. Pour le premier, on travaillera avec le réseau de $00 \mathrm{~h}$ TU disponible par telex via le réseau de veille mondiale vers $06 \mathrm{~h}$, et pour le second, au sol, on prendra les données de $06 \mathrm{~h}$ TU, qui donneront peut-être un indice d'évolution temporelle $00 \mathrm{~h}-06 \mathrm{~h}$ de la situation, et seront disponibles aussi vers $07 \mathrm{hTU}$. En effet, l'idée est de prévoir les pluies du jour $J$ (de $J$ à 06 h TU à $J+1$ à 06 h TU) à partir de la situation synoptique connue vers $00 \mathrm{~h}$, ce qui permet d'effectuer la prévision et de l'envoyer vers $8 \mathrm{~h} 30$ - 9h. La première archive constituée ne comprendra qu'une dizaine d'années (1959-68), mais il faut dire que pour certaines stations, il a même fallu aller sur le site du radiosondage les recopier manuellement ...

La question suivante a été de trouver un critère d'analogie entre deux journées, caractérisées par ces 25 valeurs de géopotentiel $700 \mathrm{mb}$... Leur corrélation n'est pas forcément un bon critère, car certaines sont proches les unes des autres et sont intercorrélées.

Et c'est là que D. Duband va faire appel à la communauté qui l'a formé. Car si l'ISUP transmet une formation solide mais classique en probabilités, elle développe aussi, notamment sous l'impulsion du Pr. Benzécri, une approche plus géométrique de l'analyse multidimensionnelle des données. Un individu $X_{i}$, caractérisé par $p$ variables $j=1, \ldots p$, peut être considéré comme un point dans un espace multidimensionnel $R^{p}$. Et un échantillon de $N$ individus $i=1, \ldots N$ constitue un nuage de points dans cet espace. On peut alors s'interroger sur la proximité de deux individus, mais aussi sur l'indépendance ou la redondance de deux variables. Il suffit de définir une distance, ou une métrique appropriée.

Plus généralement, si l'on considère un paquet de variables, on peut s'interroger sur leurs liaisons internes, les combinaisons (linéaires) ou les facteurs principaux sous-jacents, réellement indépendants statistiquement et collectant successivement le maximum de variance : on fera alors une Analyse en Composantes Principales ou ACP.

On peut aussi considérer deux paquets ou groupes de variables et analyser les liaisons intra-groupes et intergroupes: ce sera de l'Analyse Canonique, avec deux cas particuliers :

- 1 paquet ne contient qu'une variable (à expliquer) : c'est la Corrélation multiple ;

- 1 paquet ne contient qu'une variable et en plus elle est discrète : c'est de l'Analyse discriminante à 2 groupes, mais aussi de la corrélation multiple sur une variable discrète.

D. Duband donc décide de considérer d'abord les variables du géopotentiel $700 \mathrm{mb}$ aux différentes stations. Celle-ci sont irrégulièrement réparties sur le proche océan et l'Europe de l'ouest, certaines assez proches et partiellement redondantes. Il décide donc de les " condenser » et d'extraire les 7 composantes indépendantes les plus informatives grâce à une Analyse en Composantes Principales. Dans cet espace réduit (de 25 à 7 dimensions), il positionne la situation cible et recherche, autour de cette dernière, les situations qui se trouvent dans une boule de proximité au sens d'une distance euclidienne (Fig. 5). Il en extrait entre 25 et 30 et, sur cet échantillon, donc dans ce voisinage, il regarde la relation qui pourrait exister entre les précipitations relevées sur les bassins cibles et les variables secondaires non encore utilisées, comme les pressions au sol (en fait les 5 premières composantes des 11 stations sol) et les épaisseurs de la couche 1000-700 mb (sur les stations de radiosondage), car cette dernière variable indexe bien la température de l'atmosphère. Si ces corrélations fournissent, sur les journées du passé, une relation significative, il y injecte alors les variables sol observées ou prévues pour la situation cible, d'où une valeur de précipitation 


\section{GRILLE DES POINTE DE PEESSION}

\section{ALPTTUDS MOYENRE JOURNALIERE DE LA STREACE $700 \mathrm{mb}$} BN GIVER

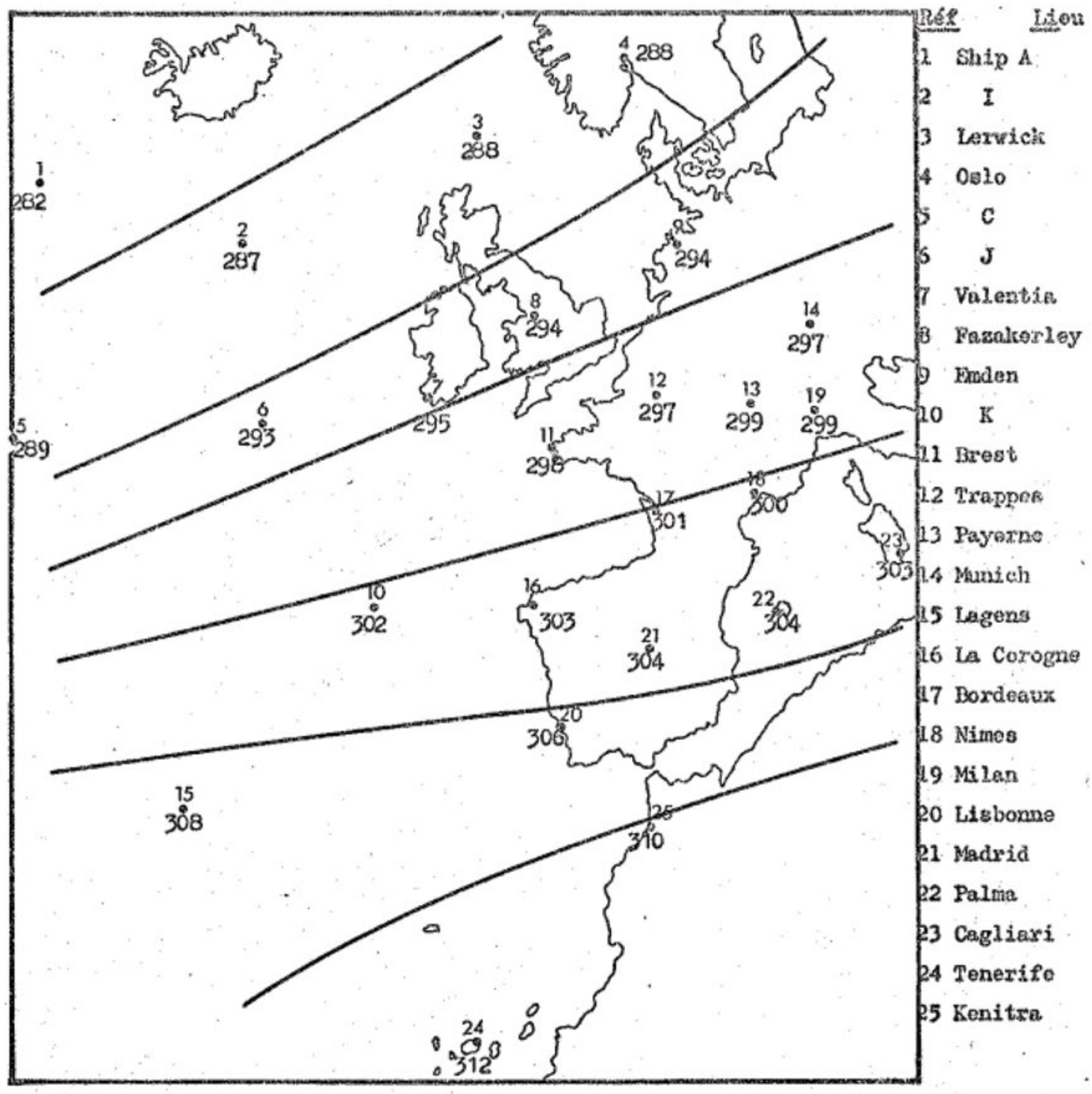

Figure 4 : Réseau des radiosondages utilisés dans les premiers travaux de D. Duband

estimée par cette corrélation et la distribution des résidus. S'il n'y a pas de relation significative, on prend alors comme prévision la distribution empirique des pluies des journées analogues pour chaque bassin considéré ...

Dans son travail initial, il se limite à une douzaine de bassins (de 500 à $2000 \mathrm{~km}^{2}$ environ), et pour minimiser les effets saisonniers, il travaille sur deux périodes : l'hiver (décembre à mars) et l'automne (septembre à novembre). Pour le jour $J$, il utilise pour caractériser la situation les radiosondages de $00 \mathrm{~h}$ TU et les données sol de $06 \mathrm{~h}$ TU. Pour $J+1$ et $J+2$, il extrapole les composantes principales respectives des champs de pression grâce à des modèles de Markov d'ordre 3 ou 4. Et cette extrapolation marchera pendant 3 ou 4 ans, le temps que le modèle EMERAUDE de la Météorologie Nationale démontre qu'à $J+1$ et $J+2$, il fait aussi bien, puis mieux, pour prévoir les champs de pression ...
En pratique, lors des premiers essais sur la CAE510, l'archive météorologique se trouvait sur une bande magnétique, que l'on balayait pour trouver la première analogue, puis on rembobinait pour chercher ensuite la seconde et ainsi de suite ..., au grand dam de l'opérateur !

Outre sa présentation à l'ISUP pour obtenir un doctorat de $3^{\text {ème }}$ cycle, cette méthode a rapidement été étendue au delà de la douzaine de bassins initiaux pour dépasser la trentaine à la fin des années 1970. De plus l'archive a progressivement été complétée, d'abord par les nouvelles années qui s'accumulaient, mais aussi en remontant dans le passé. Cette méthode a connu de nombreuses améliorations, à la fois en interne, mais aussi, comme on l'évoquera dans un chapitre suivant, dans le cadre de diverses collaborations, notamment avec le Laboratoire des Transfert en Hydrologie et Environnement (LTHE) de Grenoble. 


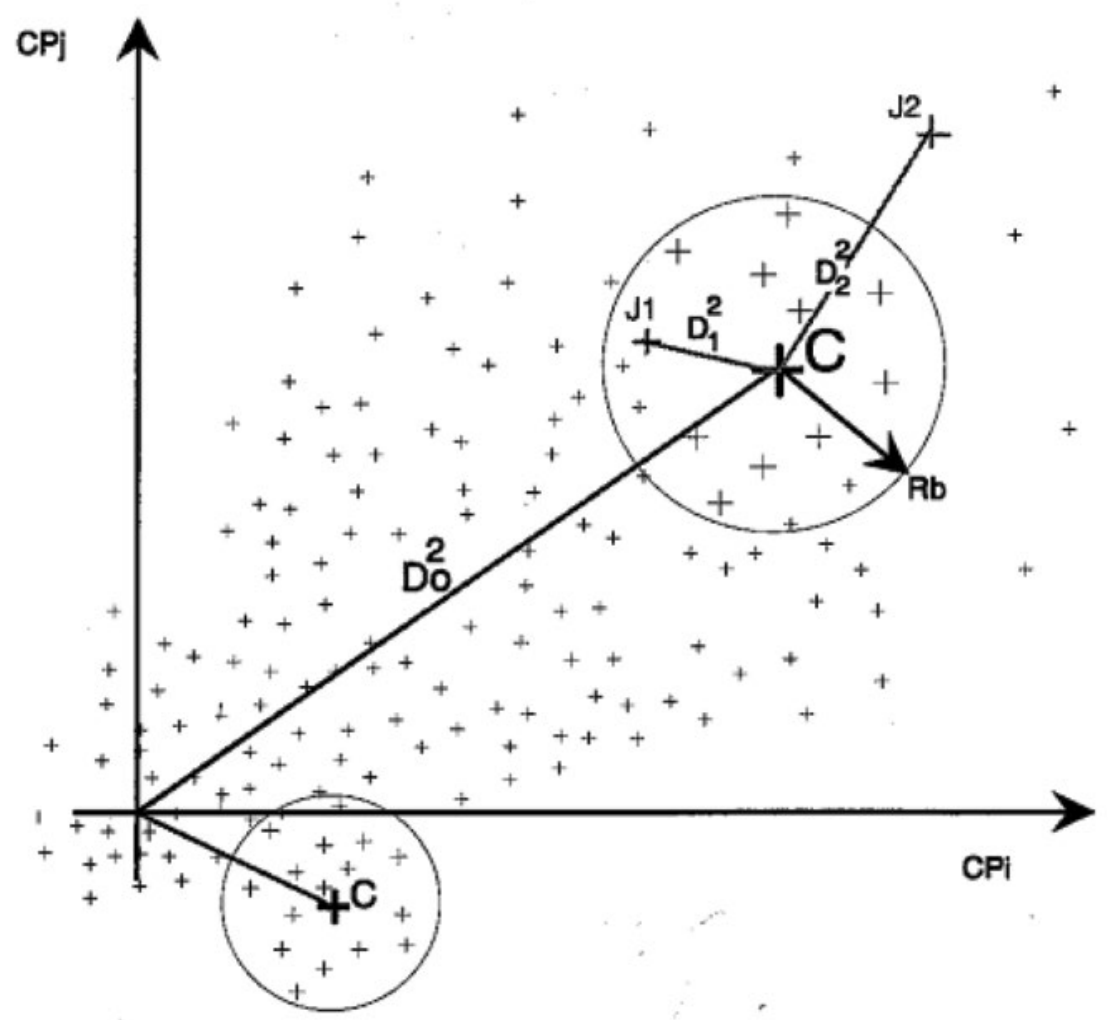

Figure 5 : Principe de sélection des analogues par boule de proximité dans l'espace des premières composantes principales des champs de pression (utilisé dans les premiers travaux de D. Duband et jusqu'en 1998).

\section{LA MÉTHODE DPFT (DIFFÉRENCE PREMIÈRE DE LA FONCTION DE TRANSFERT)}

(en anglais: ERUHDIT Excess Rainfall and Unit Hydrograph Differential Iterative Technique)

Dans les années 1977-78, D. Duband devient responsable de la prévision au sein du service Ressources en Eau, que dirige P. Guillot. Cette fois, l'effort n'est plus sur les modèles de prévision d'apports saisonniers ou hebdomadaires (car jusqu'alors, les plans de production étaient élaborés plutôt pour la semaine à venir), mais sur la prévision journalière. Il faut utiliser pour cela des modèles pluie-débits simples, mais dans lesquels la non-linéarité de la transformation pluie-débits sera prise en compte. Sur un tel sujet, le tandem P. Guillot-D. Duband se reforme et retient le schéma conceptuel classique de l'hydrogramme unitaire, dans lequel un premier module, dit " de production », décide de la part de pluie brute (tombée sur le bassin) qui sera mise à disposition de l'écoulement « rapide », tandis qu'un second module « de transfert » répartira dans le temps l'arrivée de cette pluie « efficace » à l'exutoire. Jusques là, rien de très original, et cela a été largement pratiqué et décrit, notamment par l'ORSTOM dans les ouvrages de Réméniéras (1986) ou de Roche (1963).

L'usage courant consistait jusques là à utiliser quelques pluies «unitaires », c'est à dire suffisamment violentes et rapides pour être considérées comme une impulsion « quasi » instantanée, suscitant de la part du bassin une « réponse impulsionnelle » qui donne donc la forme de son hydrogramme unitaire. Cependant, si ces conditions se rencontrent assez facilement dans les bassins tropicaux, cela est moins habituel sous nos climats où des pluies plus prolongées engendrent des réponses combinées plus complexes.
Celles-ci peuvent être vues comme la combinaison des réponses unitaires des différentes impulsions de pluies successives. De plus, celles-ci n'ont pas le même rendement, la même " efficacité » pour l'écoulement, au fur et à mesure que l'événement se développe et que le bassin se sature ...

Les auteurs voulaient d'abord que ce soit un ensemble conséquent d'évènements de crue (plusieurs dizaines, aussi bien brefs que prolongés) qui propose la réponse impulsionnelle du bassin et lui donne ainsi une certaine robustesse statistique. D'autre part, l'approche classique consiste, pour le " module de production », i.e. le module Pluie-Brute-Pluie «Efficace », à choisir une « loi » de pertes ou d'infiltration, un modèle (Horton, ou autres), bref une structure fixée a priori, paramétrée, que l'on calibre sur les données. Mais ce faisant, selon que cette partie a été choisie trop ou pas assez réactive, le calage en aval de la fonction de transfert induira des compensations qui risqueront d'interférer et de modifier la forme de la réponse impulsionnelle dans ce second module ...

D'où leur souhait de ne pas fixer a priori la structure du module de production, pas plus que la forme de la fonction de transfert, et de laisser les données fournir des éléments pour faire ces choix a posteriori. Là, l'idée géniale est d'imaginer un processus itératif convergent n'utilisant qu'un ingrédient simple : la corrélation multiple !, et un habile jeu de contraintes pour assurer la progression et la convergence vers une solution raisonnablement unique.

Par hypothèse, tous les débits (préalablement filtrés d'un débit de base) constituent des combinaisons linéaires, des convolutions de « la » réponse impulsionnelle, supposée unique, avec les pluies « efficaces » antécédentes ... Or qu'est-ce qui peut approcher, même grossièrement, ou en tout cas majorer ces pluies efficaces ... ? eh bien ! les pluies 
brutes pardi ! Donc en corrélant les débits successifs de toutes les crues mises à la queue leu leu, on va obtenir une série de coefficients de régression sur les débits de $j, j-1, j-2, \ldots j-K$ , qui donneront une idée moyenne de la forme de la réponse à une pluie en fonction des pas de temps successifs $1,2, \ldots$, $K$, où $K$ est la longueur (en pas de temps) au delà de laquelle la réponse impulsionnelle devient négligeable. Bien sûr, ces coefficients seront sensibles à l'échantillonnage, notamment à cause de la corrélation sérielle des débits. L'astuce proposée par P. Guillot et D. Duband est alors de travailler non pas sur les débits, mais sur les variations de débits d'un pas de temps à l'autre. Celles-ci sont beaucoup moins autocorrélées temporellement, moins sensibles au filtrage du débit de base, et on montre simplement qu'elles permettent d'identifier les différences premières de la fonction de transfert, que l'on peut ensuite reconstituer facilement. Bien sûr cette estimation de la fonction de transfert est sensible aux bruits numériques, aux incertitudes sur les données, d'où des oscillations qui nécessiteront un premier lissage, notamment de sa queue qui doit décroître de façon monotone. D'autre part, cette fonction de transfert est supposée conservative (i.e. tout le volume de pluie efficace est censé se retrouver dans les débits, filtrés des débits de base) : on va donc la renormer autoritairement à 1 .

Mais disposant désormais d'une première estimation de cette fonction de transfert, pourquoi ne pas considérer, crue par crue, que les débits, connus, ne sont que les transformées par cette fonction de transfert, désormais connue elle aussi !, des pluies efficaces de cet épisode ... On peut alors tenter de résoudre le problème inverse et d'identifier, de déconvoluer, crue par crue, les pluies efficaces qui, avec une telle fonction de transfert, ont donné de tels débits ... Bien sûr ce problème est souvent mal conditionné numériquement, et l'on utilisera une version de la corrélation multiple légèrement biaisée mais rendue très robuste : la régression " en crête " (Ridge regression). De plus, les résultats devront faire l'objet de quelques corrections en leur appliquant des contraintes : la pluie efficace peut-elle être supérieure à la pluie brute ? ou positive lors d'un pas de temps où il n'y a pas de pluie «mesurée »?

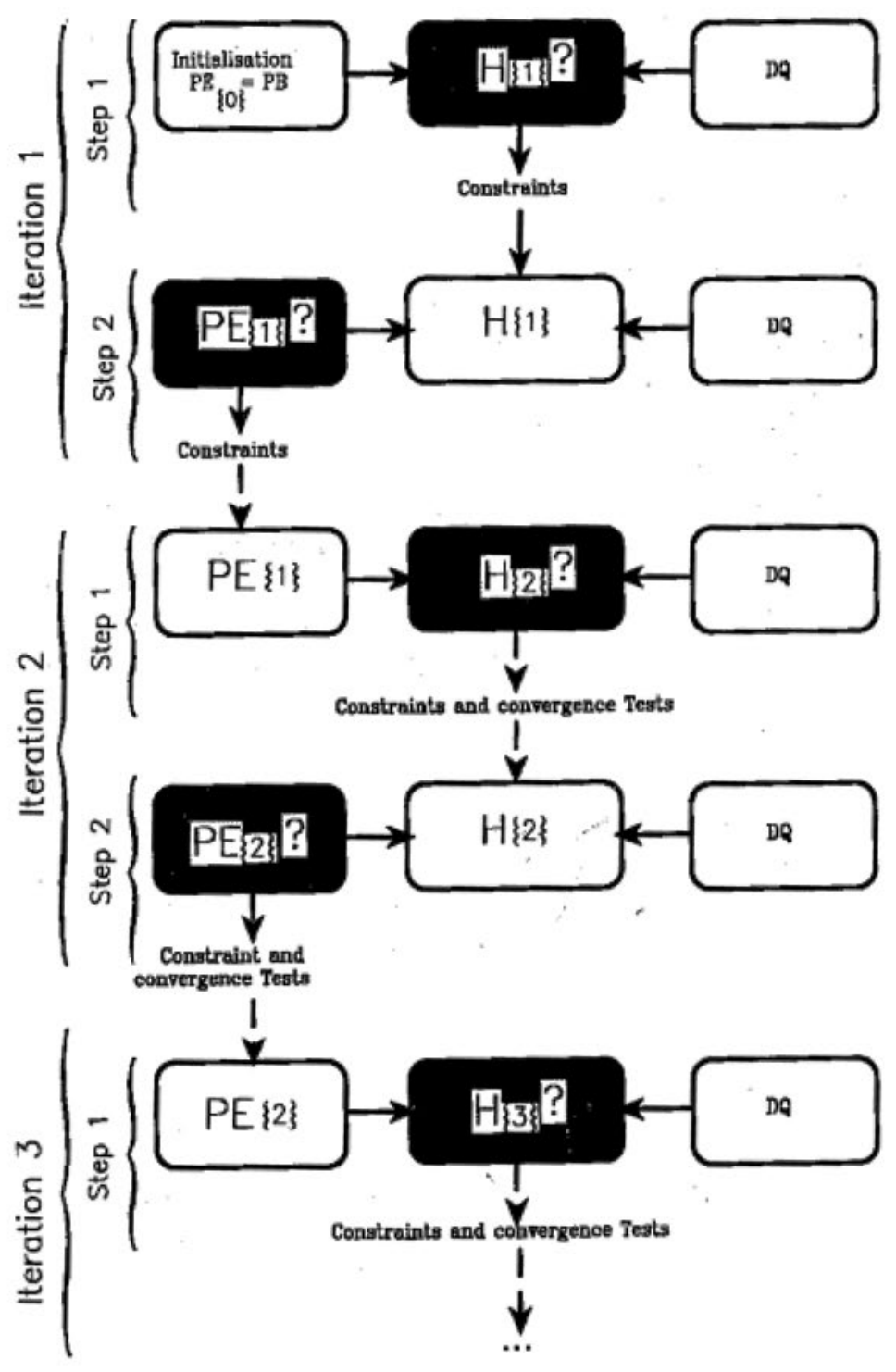

Uniknowns

( 10 be identisiod at in's step)

Figure 6 : Principe d'identification itératif alterné de la DPFT (Duband et al., 1993). 
ou présenter des oscillations alors que la pluie brute observée est monotone croissante ? ... Cela va dépendre de la confiance que l'on a dans la pluie de bassin estimée ... Mais on aboutit ainsi à un jeu de pluies efficaces candidates plus réaliste que les pluies brutes utilisées initialement ...

On itère alors le processus (Fig. 6), en les utilisant en entrée pour reconsidérer l'identification de la fonction de transfert, et ainsi de suite jusqu'à la convergence, qui nécessite en général 4 ou 5 itérations. On a alors comme résultats :

- d'une part une fonction de transfert moyenne qui nous informe sur la vitesse et l'intensité de réaction du bassin,

- et d'autre part, sur le jeu d'épisodes de crue pour lequel on avait fourni la pluie de bassin, on trouve désormais en face, à chaque pas de temps, une estimation de la fraction présumée « efficace » pour reproduire au mieux, avec la fonction de transfert ci-dessus, les débits observés.

On voit donc qu'il n'y a pour l'instant aucun modèle de production imposé ou inclus dans la méthode, et que l'on n'a pas évoqué explicitement les conditions antérieures susceptibles d'expliquer les « rendements » observés ... Ce n'est qu'ensuite que le problème se pose, et de manière complètement ouverte. P. Guillot et D. Duband choisiront ainsi, sans vraiment le documenter, un modèle assez proche du SCS, mais des versions globales de Topmodel, ou des lois d'infiltration hortonniennes ont aussi été proposées. On comprendra cependant que plus les pluies efficaces proposées par tel ou tel modèle s'éloigneront de celles identifiées, moins les performances seront bonnes ... La première publication, très détaillée, de la méthode (Guillot et Duband, 1980) montre un exemple d'application sur le Buech aux Chambons (Fig. 7).

En pratique, la mise en œuvre de cette méthode se révèle de fait un formidable exercice diagnostique pour l'hydrologue ... Pourquoi sur certaines crues, la déconvolution ne marche-t-elle pas ? Souvent, il s'agira de crue où la pluie de bassin, même correctement estimée, n'a touché qu'une partie du bassin, répondant alors avec une fonction de transfert différente de celle identifiée pour le bassin global. Une fois ce fait validé, il faudra neutraliser cet épisode. Car la
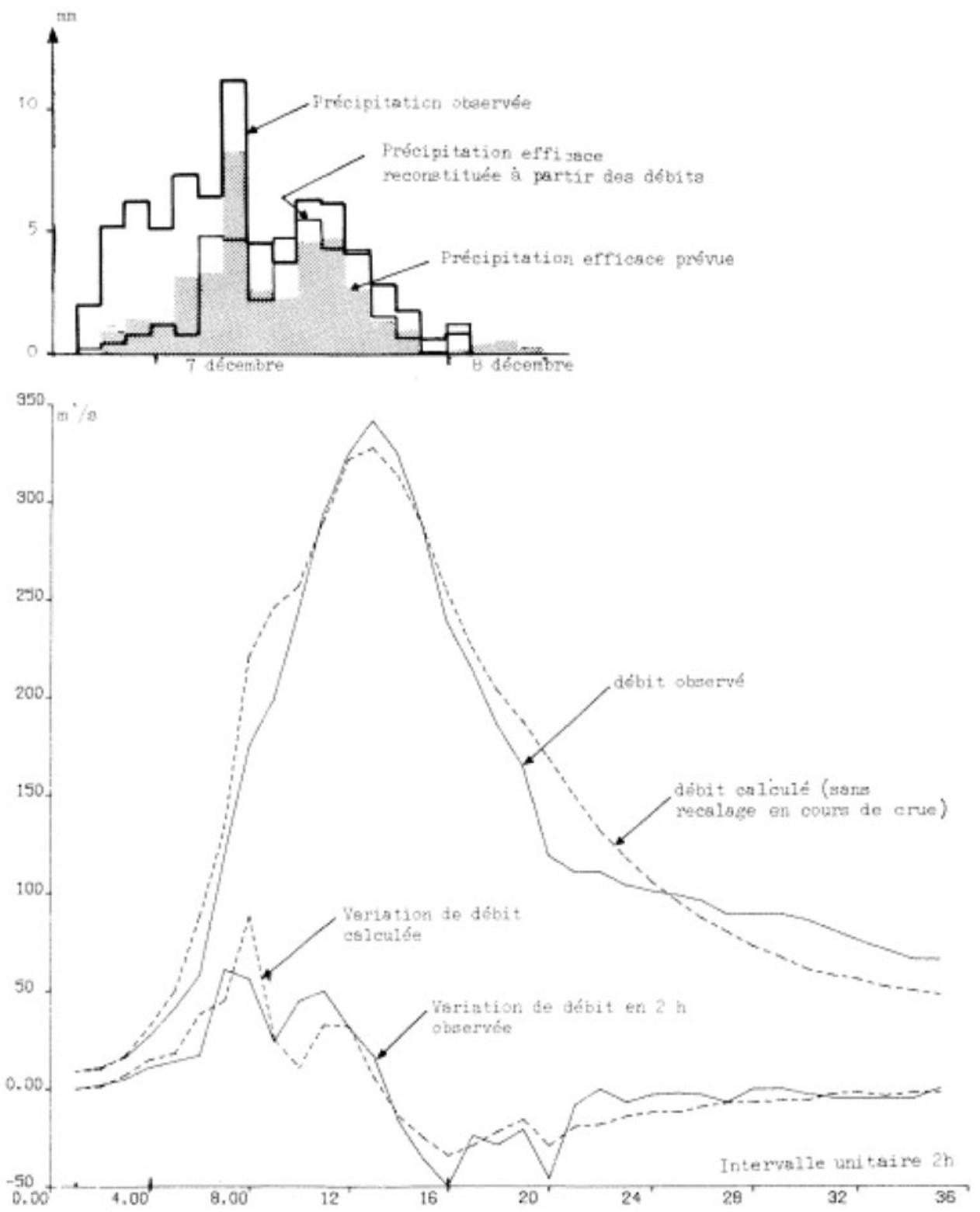

Figure 7 : Premier exemple publié d'application de la DPFT (Guillot et Duband, 1980) : Buech aux Chambons, crue-test du 8 décembre 1977. 
méthode exige, non pas, comme on l'a dit, que la pluie soit uniforme sur le bassin!, mais que sa répartition spatiale, même très contrastée, soit la répartition spatiale dominante, majoritaire lors des épisodes de crue (par exemple une pluie systématiquement plus forte sur l'amont pour des raisons d'orographie ...). Mais évidemment, dès que la pluie aura une répartition spatiale atypique, la FT moyenne sera à reconsidérer, puisque ce sera plutôt celle d'un tributaire, ou de la partie aval du bassin ...

On voit que cette méthode diagnostique constitue d'abord un formidable exercice de critique des données (sur les pluies de bassins, sur les débits, sur les répartitions spatiales -quand on les connaît-) et qu'elle suscite de nombreuses et intéressantes interrogations qui devront servir ensuite dans le choix et la conception d'un modèle opérationnel (Obled et al., 2009). Par exemple, on voit dans la figure 8a la fonction de transfert identifiée pour le Gardon à Anduze, avec une seconde bosse un peu curieuse dans la récession. Parallèlement, on donne en figure $8 \mathrm{~b}$ la distribution de l'indice topographique de Beven et Kirkby (1979) pour ce même bassin, ingrédient essentiel de TOPMODEL. Or la ressemblance est frappante, qui inclue aussi une seconde bosse, et qui reste encore à interpréter ... ! Cependant, pour appliquer facilement la méthode DPFT et accumuler les expériences, il faudrait disposer d'un outil informatique convivial et maintenu au travers des changements incessants de systèmes d'exploitation ... On verra que ce n'est pas forcément facile !
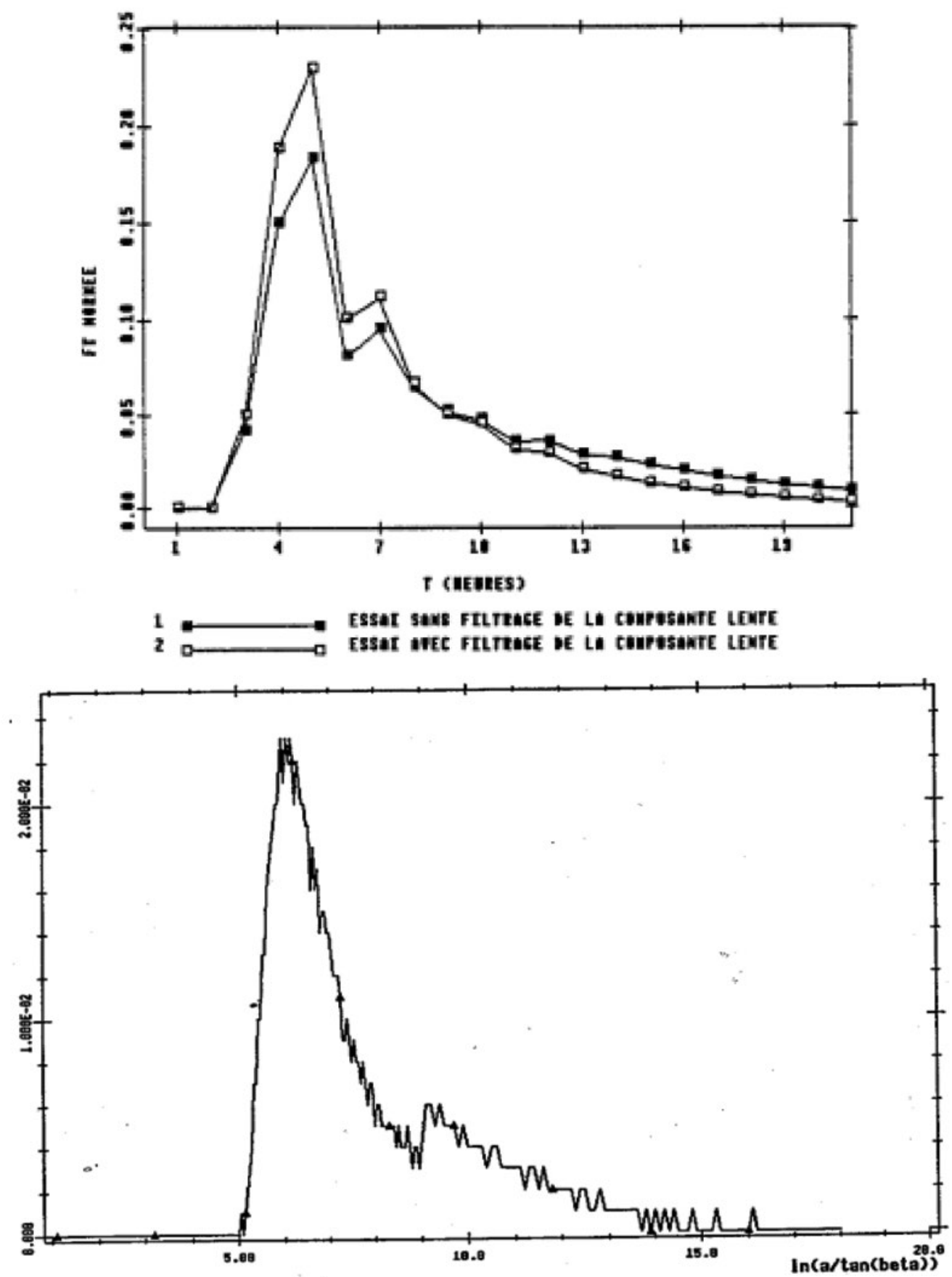

Figure 8 : a/ Fonction de transfert horaire du Gardon à Anduze (identifiée sur 35 épisodes) ; b/ Distribution de l'indice topographique (propension à la saturation) du Gardon à Anduze (repris de Obled et al., 2009). 


\section{DEVENIR ET ESSAIMAGE DES MÉTHODES INITIÉES PAR D. DUBAND}

On comprendra aussi qu'il n'était pas possible ici de parcourir tous les domaines dans lesquels D. Duband a contribué, comme par exemple, et sans prétention d'exhaustivité !, la prévision et la prédétermination des étiages, à commencer par ceux de la Loire, la prévision des épisodes de neige collante, dévastateurs pour les lignes électriques, ou les prévisions de températures pour la consommation d'énergie ... $\mathrm{Ni}$ d'évoquer l'aide et l'incitation qu'il a su apporter dans des domaines proches, comme le traitement des données d'auscultation des ouvrages. A la fin de sa carrière, il a naturellement eu un rayonnement qui a dépassé son institution d'origine, et son rôle incitateur a débordé largement l'environnement grenoblois, que ce soit à travers des enseignements, des comités de thèse, des comités de programmes de recherche ... Nous allons cependant nous concentrer sur les trois méthodes évoquées ci-dessus, car il en a vraiment été l'initiateur, il les a suivies pas à pas, même s'il a eu la chance (et l'habileté !) de ne pratiquement jamais mettre les mains dans leur programmation ... On constatera alors que leur devenir va varier à la fois selon l'époque où la méthode a été proposée, mais aussi selon les contextes scientifique et industriel du moment où l'on se placera.

Pour la méthode du Gradex par exemple, le développement s'est fait essentiellement en interne à EdF. Il s'agit d'une méthode de dimensionnement essentielle pour la sécurité des ouvrages, dans laquelle la responsabilité de l'entreprise est engagée. Le débat scientifique a lieu lui aussi quasiment " en interne », ou en public à la Société Hydrotechnique de France (mais si l'on considère le poids qu'y représentait EdF à l'époque ...), et avec deux écoles qui tendent à apparaître, entre DTG-Grenoble et DER-LNH Chatou. Certes des extérieurs tenteront bien d'y contribuer aussi, notamment au CTGREF (Michel et Oberlin, 1972), mais ils seront peu entendus. Ils tenteront de récidiver dans les années 1990 avec la méthode AGREGEE, qui se veut dans la mouvance $\mathrm{du}$ Gradex mais plus progressive dans les extrapolations (Margoum 1992 , Margoum et al. 1994) sans pour autant convaincre les «pères » de la méthode. Ceux-ci ont plutôt recherché une reconnaissance du monde de l'ingénierie, y compris à l'international, face aux approches américaines de type PMP-PMF (Probable Maximum Precipitation-Probable Maximum Flood), largement préconisées aux USA mais aussi dans les grands projets internationaux. La méthode du Gradex a cependant été la méthode de référence en France jusqu'à la fin des années 1990-2000. Elle a suscité un grand intérêt dans les pays voisins, et a été consacrée lors du congrès des grands barrage de San Francisco (Duband et al., 1988). Elle a fait l'objet d'une publication bilingue préparée par un groupe de travail du CFGB (1994). Ce n'est que récemment qu'elle a connu des modifications substantielles avec la méthode SCHADEX (Garavaglia, 2011 ; Paquet et al., 2013). Tout d'abord, on y reconnaît que la loi des pluies, et donc de leurs extrema, est différente selon les types de temps. La loi résultante du maximum annuel est donc une combinaison de ces lois pondérées par la fréquence d'apparition de chaque type de temps. Ensuite, pour passer au débit, et au lieu du modèle simpliste type SCS, on utilise une approche Monte-Carlo avec un modèle pluie-débit plus élaboré, le modèle MORDOR, développé pour la prévision opérationnelle.

Pour l'approche par analogues, le contexte était complètement différent. L'enjeu n'était pas stratégique pour l'entreprise, même si la prévision peut conduire à des gains cumulés substantiels. De plus, l'initiative de cette approche provenait de D. Duband lui-même, et non d'une demande institutionnelle ou hiérarchique ... Elle lui avait permis d'en tirer une thèse, certes honorifique pour le service, mais cela ressemblait un peu trop à une démarche de recherche ... or la Recherche, c'était la DER de Chatou ou Clamart. La DTG devait se limiter à du développement de méthode et de techniques instrumentales au service de la Production Hydraulique, et ne pas empiéter sur le domaine de la recherche exploratoire ... Afin de contourner ces sensibilités parfois ombrageuses, F. Lugiez avait déjà imaginé, dès 1962, une tactique : l'externalisation, vers l'université de proximité ! Ce fut la thèse de A. Poggi (1966) sur le bilan thermique de la neige. P. Guillot, intéressé à se faire une idée sur les modèles hydrologiques (qu'il vilipendait par ailleurs !), avait fait de même en suscitant la thèse de Ch. Obled (1971). D. Duband, face au même dilemme, et qui ressentait peut-être une certaine impression de solitude et le besoin de trouver ailleurs une occasion de dialogue, s'empressa de suivre le même chemin ! Après avoir suivi, par intérêt amical, nos travaux (Bois et Obled 1976) sur la prévision d'avalanches par des techniques d'analyse discriminante, il nous a suggéré la possibilité d'une approche par analogues, dans un espace de variables appropriées ... Certes définir la similitude de deux situations nivométéorologiques vis à vis du risque avalanche n'est pas chose aisée ..., non plus que le critère de distance à utiliser. Toujours est-il que cela a suscité des débats animés mais a finalement bien marché (Obled et Good, 1980), et que l'approche développée en 1978 a été utilisée jusqu'à tout récemment au Centre de Prévision Neige et Avalanches (SLF) de Davos.

Au début des années 1980, cette fois dans le cadre du programme PIRDES pour le développement de l'énergie solaire, nous avons adapté la méthode pour la prévision des durées d'insolation (Thalamy, 1981). D. Duband avait mis à notre disposition l'archive météorologique d'EdF, et en contrepartie, nous le faisions participer à nos avancées et à nos doutes ... Nous prenions même des stagiaires (notamment à l'Ecole Nationale de la Météorologie) pour tester des variantes ou des idées nouvelles susceptibles d'être implantées dans la version opérationnelle à EdF. Cette confiance est allée jusqu'à l'accompagnement de la thèse de S. Guilbaud (1997), où nous avons reconsidéré la méthode en passant de l'archive des radio-sondages à des grilles de données réanalysées, et en changeant le critère « de distance » euclidienne pour un critère plus météorologique mesurant l'analogie « de forme » de la circulation (Teweles-Wobus). Convaincu par les résultats, D. Duband décidait d'implanter cette nouvelle version. Mais après quelques mois, un doute s'installa, très nuisible à notre crédibilité ... Jusqu'à ce que l'on trouve qu'en la reprogrammant, une malheureuse interversion d'indices faisait que l'algorithme cherchait en regardant selon l'axe Est Ouest des analogues orientées Nord-Sud ... Ouf ! tout rentra dans l'ordre, et les performances se retrouvèrent à la bonne hauteur ... !

Dans les années qui suivirent, D. Duband, bien que très pris par de nouvelles responsabilités, ne cessa pas de nous encourager, d'abord en soutenant notre participation au programme RIO du Ministère de l'Environnement. Il a suivi attentivement les développements réalisés sur la méthode des analogues au cours de plusieurs thèses, à Grenoble (Djerboua 2001, et Bontron 2004), puis au Cemagref à Lyon (Ben Daoud, 2010 ; Ben Daoud et al., 2011), avec une implémentation et une remise en forme à la Compagnie Nationale du Rhône (Fig. 9), puis à nouveau à Grenoble (Marty, 2010 ; Marty et al., 2011), et encore à Lausanne (Horton, 2012 ; Horton et al., 2012), 
toujours dans une optique de prévision. Elle a aussi été appliquée et développée, notamment dans ses aspects interprétation météorologique et aérologique, par Gibergans-Báguena et Llasat (2007), à l'Université de Barcelone. De plus, elle est désormais utilisée en descente d'échelle pour désagréger les scénarios climatiques (Lafaysse et al., 2013) ... Bien sûr, les apôtres du déterminisme à tout crin n'ont cessé, depuis les années 1970, de la condamner à une obsolescence prochaine, tant les modèles météorologiques allaient faire de progrès ... Ce qu'ils firent d'ailleurs, mais non pas tant sur les précipitations que sur les prédicteurs synoptiques et aérologiques utilisés pour l'analogie, et cette dernière, à chaque amélioration, s'empresse d'en faire son profit ! C'est ainsi que les réanalyses nous offrent désormais des archives remontant à 1947 (NCEP-NCAR) voire au début du XXème siècle pour les données sol ... Et les techniques d'optimisation et de " data mining " permettent aujourd'hui une exploration beaucoup plus exhaustive des données ... Cela explique que, quarante ans après sa proposition par D. Duband, l'approche par analogues, du moins sur les pas de temps que les archives lui permettent, relève encore vaillamment le défi avec des modèles bien plus gros qu'elle ....!

Pour la DPFT, par contre, le bilan sera un peu plus contrasté. A l'époque où elle est apparue (Guillot et Duband, 1980), ses auteurs se sont trouvés de plus en plus pris par les responsabilités d'un service qui avait considérablement grossi. D. Duband en a assuré le suivi mais il en a externalisé le développement, puisqu'elle a fait l'objet d'un DEA puis de plusieurs thèses (Versiani 1984, J. Nalbantis 1987, Y. Rodriguez 1989, D. Sempere-Torres 1990). Grâce à celles-ci, les algorithmes ont été considérablement améliorés, validés aussi sur données artificielles simulées, et la méthode de double identification (fonction de transfert et séries de pluies efficaces) bien stabilisée (Duband et al., 1993 ; Nalbantis et al., 1995). Malheureusement, on peut dire qu'ensuite, sa diffusion a fait l'objet de deux erreurs de communication ...

Tout d'abord, elle a été présentée comme "le modèle » DPFT ..., alors que justement elle n'en était pas un ! En fait, comme on l'a indiqué, il s'agissait plutôt d'une méthode diagnostique, d'une aide à la modélisation, proposant une fonction de transfert relativement robuste et des séries efficaces indicatives, susceptibles d'orienter le choix d'un " modèle de production ", d'infiltration ou d'une fonction de perte, et éventuellement d'aider à sa calibration. Mais le fait qu'EdF ait " encapsulé » dans son logiciel deux ou trois fonctions candidates renforçait cette impression de "boite noire » presse-bouton et capable de tout faire ... D'autre part, à l'époque, le début des années 1990, la notion d'entreprise publique ne faisait plus rêver ... et la DTG, dont on envisageait même l'externalisation, avait reçu pour directive de valoriser ses produits et services vers le monde de l'ingénierie. De ce fait, D. Duband, désormais à la tête du Service Ressources en Eau, avait décidé que le logiciel serait distribué seulement sous forme exécutable, et à titre onéreux pour les bureaux d'études ... Il s'écartait ainsi de la démarche ouverte que ses prédécesseurs et lui-même avait privilégiée jusques là, en mettant gratuitement à disposition les méthodes et bien souvent les données, en échange de collaborations et de retour sur les améliorations apportées, et pour le plus grand bien de la collectivité ... Enfin le logiciel réalisé, bien que largement suffisant techniquement pour les besoins propres d'EdF, n'offrait pas une convivialité et une transférabilité suffisante permettant son activation facile par un non-initié. De ce fait, la méthode aujourd'hui n'est presque plus utilisée, en dépit de son intérêt diagnostique indéniable, même si le LCPC (IISTAR -Nantes) a tenté d'en maintenir une version. Celle-ci mériterait certainement une remise à niveau et une diffusion plus accessible ... Mais cela montre aussi que même les grands hommes ont leurs faiblesses, et que même les bonnes idées peuvent disparaître ... !

Le 7 Juin 2011, Daniel Duband a dû annuler en urgence une réunion préparatoire à un séminaire SHF intitulé «Passeurs-Traducteurs » (Water System Science and Policy Interface). Celui-ci était destiné à « faire un état des lieux préliminaire sur la médiation (diffusion, concertation) des savoirs, la diffusion de l'information au sein d'une société en attente : comment passer une information scientifique et technique vers les acteurs publics, vers les décideurs, les élus ...? ?. Cela démontrait une fois de plus son souci de la transmission des savoirs accumulés ... Malheureusement, moins

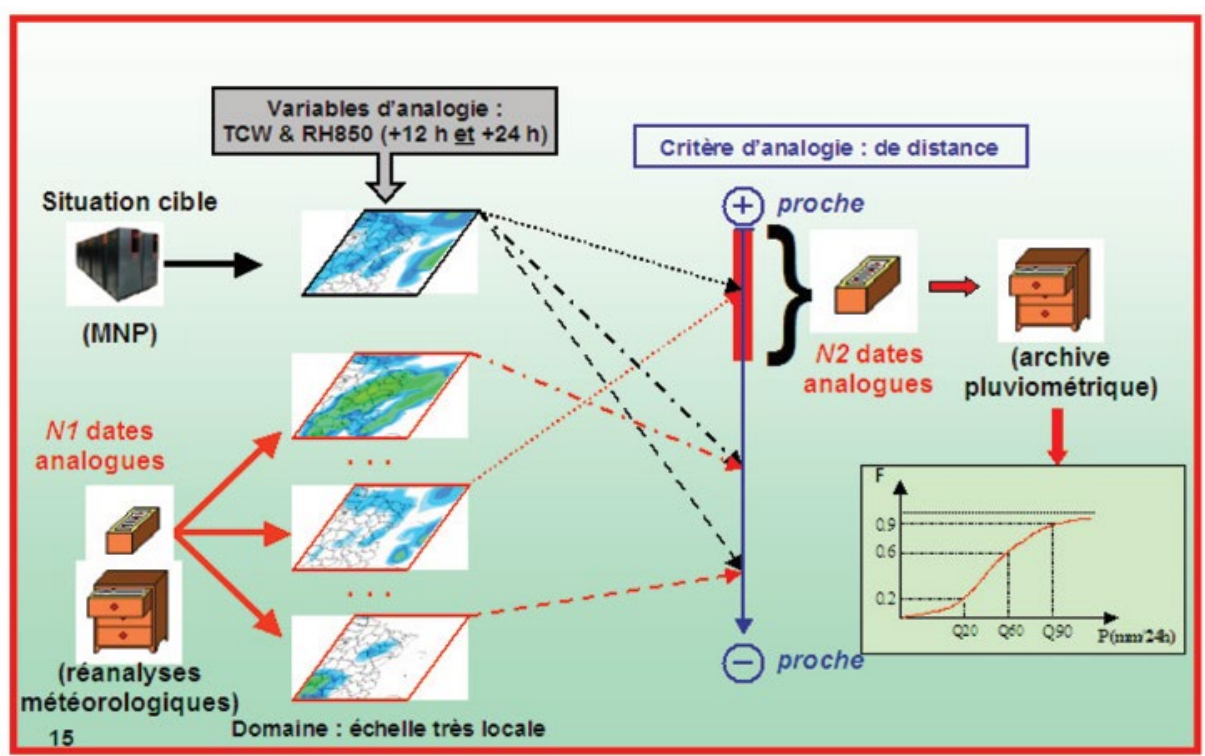

Figure 9 : Exemple récent d'implantation de la méthode des analogues, avec deux niveaux successifs d'analogie, le premier sur la forme des champs de pression et le second sur des variables d'humidité. (Ben Daoud, 2010). 
d'un mois après, il nous quittait ... Nous étions nombreux à avoir des idées, des projets, des travaux, des tas de choses en cours dont on se disait ... « qu'il faudrait trouver un moment pour en parler avec D. Duband ...». Malheureusement, ce moment, nous ne le trouverons plus, et c'est peu dire que Daniel Duband n'a pas fini de nous manquer ...

\section{Remerciements}

Je remercie tous ceux qui ont accepté de relire et d'alimenter ce texte avec leurs souvenirs. D'abord mon collègue et ami $\mathrm{Ph}$. Bois avec qui j'ai partagé pendant des années cette proximité avec le Service Ressource en Eau de la DTG, mais aussi des proches collaborateurs de D. Duband, comme F. Hirrien, F. Argelès, et M. Jacob, et, plus récemment, R. Garçon, P. Tourasse, qui ont travaillé à ses côtés pendant des années. Enfin M. Lang, de l'Irstea, a soigneusement révisé ce texte et m'a beaucoup aidé dans sa mise en forme finale.

\section{REFERENCES}

Ben Daoud A. , E. Sauquet M. Lang G. Bontron 1 C. Obled (2011) - Precipitation forecasting through an analog sorting technique: a comparative study. Advances in Geosciences (ADGEO). 29 : 103-107

Ben Daoud A. (2010) - Améliorations et développements d'une méthode de prévision probabiliste des pluies par analogie. Application à la prévision hydrologique sur les grands bassins fluviaux de la Saône et de la Seine. Thèse Univ. Grenoble

BERNIER J. (1956) - Sur l'application des diverses lois limites des valeurs extrêmes au problème des débits de crue. La Houille Blanche. 5

Bernier J. (1959) - Comparaison des lois de Gumbel et de Fréchet sur l'estimation des débits maxima de crue. La Houille Blanche. 1

Beven K.J. Et M.J. Kirkby (1979) - A physically-based, variable contributing area model of basin hydrology. Hydrol. Sci. Bull. 24 (1) : 43-69

Bois Ph. Eт CH. Obled (1976) - Prévision des avalanches par des méthodes statistiques: Aspects méthodologiques et opérationnels. La Houille Blanche. 6-7 : 509-531

Bontron G. (2004) - Prévision quantitative des précipitations : adaptation probabiliste par recherche d'analogues. Utilisation des réanalyses NCEP/NCAR et application aux précipitations du sud-est de la France. Thèse INP-Grenoble

CFGB (1994) - Les crues de projet des barrages : méthode du GRADEX . Design Flood Determination by the Gradex Method. Bulletin du Comité Français des Grands Barrages. 2 : 96p

Coutagne A. (1948) — Météorologie et hydrologie. Etude générale des débits et des facteurs qui les conditionnent. $2^{\circ}$ partie : les variations de débit en période non influencée par les précipitations. Le débit d'infiltration (corrélations fluviales internes). La Houille Blanche. 5 : 416-436

Djerboua (2001) — Prédétermination des pluies et crues extrêmes dans les Alpes franco-italiennes. Prévision quantitative des pluies journalières par la méthode des analogues. Thèse INP-Grenoble

Duband D., Michel C., Garros H. Et Astier J. (1988) Evaluation des Crues Extrêmes et de la Crue de Projet par la Méthode du Gradex. Estimating Extreme value Floods and the Design Flood by the Gradex Method. Commission Internationale des Grands Barrages. XVIe Congrès des grands Barrages San Francisco Proceedings. IV : 1009-1047

Duband D., Obled Ch. Et Y. Rodriguez (1993) - Unit Hydrograph Revisited: An Alternate Iterative Approach for
U.H. and Effective Precipitation Identification. J. of Hydrology. 150 : $115-149$

FERRY S (1949) - Les méthodes d'estimation de l'enneigement dans les bassins de montagne et leur utilisation à la prévision des apports de printemps et d'été. Union internat. des producteurs et distributeurs d'énergie électrique. Congrès de Bruxelles 19-24 Sept

Ferry S Et F. Lugiez (1951) - Prévision d'apports durant la période de fusion des réserves nivales. UGGI, Assemblée Générale de Bruxelles, publi. AIHS. 34

FERRY S. (1955) - L'utilisation des méthodes statistiques à EdF pour la prévision des apports dans les réservoirs- Examen des résultats obtenus. Séance de 1'ITAP organisée par le Comité Technique. Revue de Statistique Appliquée. 19

Garavaglia F. (2011) - Méthode SCHADEX de prédétermination des crues extrêmes. Méthodologie, applications, études de sensibilité. Thèse de l'Université de Grenoble

Gibergans-Baguena J. Et M.C. Llasat (2007) - Improvement of the analog forecasting method by using local thermodynamic data. Application to autumn precipitation in Catalonia. Atmospheric Research. 86 : 173-193

Guilbaud S. (1997) - Prévision Quantitative des Précipitations Journalières par une méthode Statistico-Dynamique de Recherche d'Analogues. Application à des Bassins du Pourtour Méditerranée. Thèse INP-Grenoble. 386p

Guillot P. (1963) — La probabilité du débit maximum annuel et ses relations avec la loi de distribution des débits journaliers. Congrès A.I.R.H. Londres, sept 1963

Guillot P. (1969) — La prévision hydrologique. EDF, DTG Grenoble

Guillot P. Et D. Duband (1967) — La méthode du Gradex pour le calcul de la probabilité des crues à partir des pluies. IAHS publ. 84 : 560-569

Guillot P. Et D. Duband (1980) — Une méthode de transfert pluiedébit par régression multiple. Oxford Symposium on Hydrological forecasting - Prévisions hydrologiques, April. Actes du Colloque d'Oxford, Arpil 1980, IAHS-AISH Publ. 129 : 177-184

Gumbel E. J. (1954) - Statistical Theory of Extreme values and some practical applications. Nat. Bureau of Standards. Applied Math. Series. 33

Gumbel E. J. (1956) - Méthode graphique pour l'analyse des débits de crues. La Houille Blanche. 5

HorTon P. (2012) - Améliorations et optimisation globale de la méthode des analogues pour la prévision statistique des précipitations. Développement d'un outil de prévision et application opérationnelle au bassin du Rhône à l'amont du Léman. Thèse de l'Université de Lausanne, Faculté des géosciences et de l'environnement

Horton P., Jaboyedoff M., Metzger R., Obled C. Et R. Marty (2012) - Spatial relationship between the atmospheric circulation and the precipitations measured in the Swiss Alps by means of the analogue method. NHESS. 12 : 777-784

Kendall M.G Et Stuart A. (1963) - The Advanced Theory of Statistics, 3 volumes, Charles Griffin, London, Gème édition

LABAYE G. (1956) - Le problème des évacuateurs de crues de Serre Ponçon: essai de détermination d'un optimum économique. Revue de Statistique Appliquée. 4(3) : 47-66

Lafaysse M., Hingray B., Gailhard J., Mezghani A. (2013) Internal variability and model uncertainty components in a multimodel multireplicate ensemble of hydrometeorological predictions. Water Ressources Research (in revision)

LugieZ F. (1954) - Un cas particulier de prévision des apports : l'Isère à Tignes. 3ème journées de l'Hydraulique Alger 12-14 avril, publication $\mathrm{SHF}$

Lugiez F. Et P. Guillot (1960) - Dix années de prévision d'apports à Electricité de France. Assemblée générale UGGI Helsinki 
Lugiez F., Plancher C, Guillot P. Et Duband D. (1963) - La capacité de rétention du sol, problème majeur pour la prévision des débits à court terme d'après les averses. U.G.G.I, Assemblée générale de Berkeley, publication de l'AISH. 63

Margoum M. (1992) - Estimation des crues rares et extrêmes : le modèle Agregee. Conceptions et premières validations - Thèse de doctorat, Ecole des Mines de Paris, Cemagref Lyon, GIS Hydrologie Friend-Amhy. 252p

Margoum M., Oberlin G., Lang M., Weingartner R. (1994) Estimation des crues rares et extrêmes : principes du modèle Agregee. Hydrologie Continentale. 9 (1) : 85-100

MARTY R. (2010) - Prévison hydrologique d'ensemble adaptée aux basins à crue rapide. Elaboration de prévisions probabilistes de précipitations à 12 et $24 \mathrm{~h}$. Désagrégation horaire conditionnelle pour la modélisation hydrologique. Application à des bassins de la région Cévennes-Vivarais. Thèse de l'Université de Grenoble

Marty R., Zin I., Obled Ch., Bontron G., A. DJerboua (2012) Towards real-time daily PQPF by an analog sorting approach. Application to flash flood prone catchments. J. of Applied Meteorology and Climatology. 51 (3) : 505-520

Michel C., OBERLIN G. (1972) — L'application de la méthode du gradex à l'estimation des crues de faible fréquence. CEMAGREFCTGREF, Division Hydrologie-Juillet 1972. 19 p

Morlat G. (1951) - Note sur l'estimation des débits de crues. La Houille Blanche. B : 663-681

Morlat G. (1956) — Les lois de probabilité de Halphen. Revue de Statistique Appliquée. 4(3) : 21-46

Nalbantis I., Obled Ch. Et Y. Rodriguez (1995) - Unit Hydrograph and Effective Precipitation Identification. $J$. of Hydrology. 168 : 127-157

NALBAntis J. (1987) - Identification de modèles pluie-débit du type hydrogramme unitaire : développement de la méthode DPFT et validation sur données simulées avec et sans erreur Thèse INPG

OBled CH. (1971) - Modèles mathématiques de la fusion nivale. Recherche d'une prévision des risques d'avalanches. Université Scientifique et Médicale de Grenoble
Obled Ch. , I. Zin B. Hingray (2009) - Choix des pas de temps et d'espace pour des modèlisations parcimonieuses en hydrologie des crues. La Houille Blanche. 5 : 81-87

Obled Ch. Eт W. Good (1980) - Recent developments in Avalanche Forecasting by statistical techniques: a methodological review and some applications to the Parsenn area (Davos-Switzerland). J. of Glaciology. XXV(92) : 315-346

Paquet E., Garavaglia F., Garcon R., Gailhard J. (2013) - The SCHADEX method : a semi-continuous rainfall-runoff simulation for extreme flood estimation. J. Hydrology. 495 : 23-37

Poggi A. (1966) - L'évolution de la neige déposée à moyenne altitude, Thèse de la faculté des sciences de l'université de Grenoble. $38 \mathrm{p}$

Remenieras G. (1986) - L'hydrologie de l'ingénieur . Paris, Eyrolles, $2^{\circ}$ ed. $456 \mathrm{p}$

Roche M. (1961) - Hydrologie de Surface . Paris, Gauthier-Villars, Orstom. $430 \mathrm{p}$

RODRIGUEZ Y. (1989) - Modélisation pluie-débit par la méthode DPFT : développements de la méthode initiale et extension à des cas bi-entrées. Thèse INP-Grenoble

SEMPERE-TORRes (1990) - Calcul de la lame ruisselée dans la modélisation pluie-débit : limitations des approches globales et introduction simplifiée de la topographie et de la variabilité spatiale des pluies. Thèse INP-Grenoble

Soll Conservation Service (1972) - National Engineering Handbook Vol. 4 Hydrology. US Govern. Printing Office, US Dept of Agriculture

Soil Conservation Service (1973) - A method for estimating volume and rate of runoff in small watersheds. Techn. Paper, US Govern. Printing Office, US Dept of Agriculture. 149

Thalamy J. (1981) — Etude de quelques situations météorologiques ayant provoqué des crues sur les Cévennes. Prévision de durée d'insolation par la méthode des plus proches voisins. Rapport de stage de fin d'Etudes de l'Ecole de la Météorologie Nationale

VERSIANI B. (1984) - Modélisation de la relation pluie-débit pour la prévision de crues. Thèse INP-Grenoble 